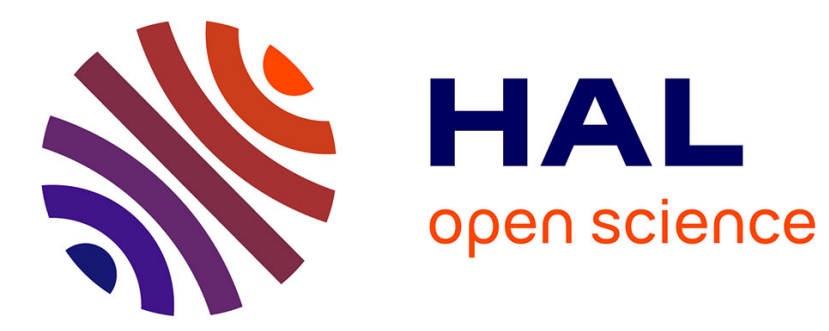

\title{
Lieux d'échanges et espaces publics en Gaule à La Tène finale
}

Thomas Hutin

\section{To cite this version:}

Thomas Hutin. Lieux d'échanges et espaces publics en Gaule à La Tène finale. Archimède: archéologie et histoire ancienne, 2016, 3, pp.135-149. hal-01587243

\section{HAL Id: hal-01587243 \\ https://hal.science/hal-01587243}

Submitted on 13 Sep 2017

HAL is a multi-disciplinary open access archive for the deposit and dissemination of scientific research documents, whether they are published or not. The documents may come from teaching and research institutions in France or abroad, or from public or private research centers.
L'archive ouverte pluridisciplinaire HAL, est destinée au dépôt et à la diffusion de documents scientifiques de niveau recherche, publiés ou non, émanant des établissements d'enseignement et de recherche français ou étrangers, des laboratoires publics ou privés. 


\section{DOSSIER THÉMATIQUE : DES FOSSÉS ET DES REMPARTS. ENCEINTES ET SITES FORTIFIÉS DU RHIN SUPÉRIEUR ENTRE PROTOHISTOIRE ET MOYEN ÂGE}

\section{Olivier BUCHSENSCHUTZ}

Avant-propos. Des enceintes en terre anhistoriques à Google Earth

8 Lizzie SCHOLTUS

Histoire de la recherche dans le bassin de Saint-Dié-des-Vosges

20 Maxime WALTER

Les sites de hauteur du massif vosgien. Actualisation des données et modalités d'implantation

37 Jean-Jacques SCHWIEN

Chateaux et enceintes des Vosges du Nord. Topographie et longue durée

49 Anne-Marie ADAM

La palissade dans tous ses états : I'enclos du Britzgyberg (Illfurth, Haut-Rhin) et autres aménagements palissadés dans les habitats du premier âge du Fer

60 Clément FÉLIU

L'enceinte inférieure du Frankenbourg (67) et les remparts à poteaux frontaux de la fin de l'âge du Fer dans l'espace du Rhin supérieur. Pour une révision de la typologie des Pfostenschlitzmauern

74 Jacky $\mathrm{KOCH}$ et Thomas FISCHBACH

Enceintes de hauteur en pierres et formes « primitives » de châteaux ? L'exemple du Bernstein

87 Adrien VUILLEMIN

Les enceintes urbaines en moyenne Alsace (1200-1850)

102 Jean-François PININGRE

Les enceintes de l'âge du Bronze et du premier âge du Fer en Franche-Comté. Un bilan des recherches

124 Clément FÉLIU et Jean-Jacques SCHWIEN

Conclusion. Nouvelles perspectives sur les enceintes du Rhin supérieur

\section{ACTUALITÉ DE LA RECHERCHE : ARCHÉOLOGIE DES RÉSEAUX}

\section{Claire CAMBERLEIN}

Les réseaux en archéologie : approche historiographique et interdisciplinaire

\section{Thomas HUTIN}

Lieux d'échanges et espaces publics en Gaule à La Tène finale

150 Streeve GENTNER

Économie du fer et voies de communication, de l'abattage du minerai à la distribution du métal : I'exemple du nord de la Forêt-Noire au Ve siècle av. J.-C.

169 Loup BERNARD et Rémy WASSONG

Du Danemark au Fossé rhénan. Un siècle d'analyse des voies de communications protohistoriques : évolution des méthodes et mise en commun des données

184 Steeve GENTNER et Rémy WASSONG

Conclusion. L'archéologie des réseaux : une thématique aux multiples facettes

\section{VARIA}

187 Fábio VERGARA CERQUEIRA

To march in phalanx, to jump with weights, to tread the grapes, to knead the bread. What is the aulos for?

206 Hermann AMON

Les supra-commandements comme solution à la crise militaire du III siècle de l'Empire romain sous Philippe l'Arabe et Gallien

218 Martina BONO

Il processo di Cremuzio Cordo in Dio LVII, 24, 2-4

\section{LA CHRONIQUE D'ARCHIMÈDE}

228 Frédéric COLIN (éd.)

La Chronique d'Archimède. Bilan des activités scientifiques 2015-2016 de I'unité mixte de recherche 7044 


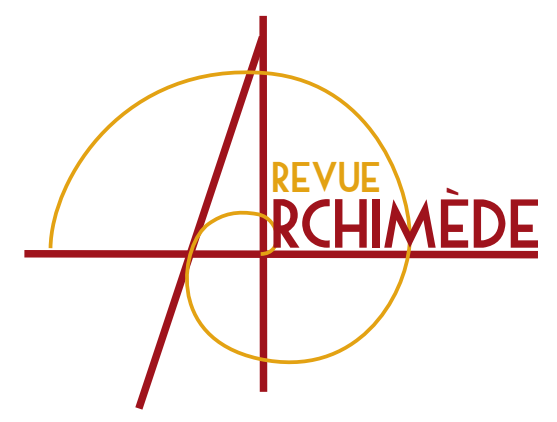

\section{LIEUX D'ÉCHANGES ET ESPACES PUBLICS EN GAULE À LA TĖNE FINALE}

Si pour les périodes antiques la question des marchés construits est une problématique récurrente, la situation est bien différente pour le monde celtique. Pendant longtemps, les réflexions portées sur les espaces de vente étaient en effet reléguées au second plan des publications scientifiques. Ces trois dernières décennies, les fouilles réalisées sur plusieurs grands oppida, et autres agglomérations, ont peu à peu révélé toute la complexité des grands centres urbains du second âge du Fer. C'est à ce titre que les opérations archéologiques effectuées sur le plateau de Corent ont permis de mettre au jour un vaste ensemble sur cour dont la vocation artisanale et commerciale ne fait aucun doute. Structuré et optimisé pour la vente de marchandises variées, cet aménagement répond parfaitement à l'image que I'on peut se faire du marché en tant qu'ouvrage architectural. Malgré l'accumulation grandissante des données de terrain, aucune analogie aussi représentative n'a pu être repérée par les fouilles. Quelques complexes sur cour ont bien été identifiés dans l'ouest et le centre-est de la France, mais aucun dont la nature et la fonction ne puissent être aussi significative. En revanche, les découvertes récentes de grandes places pourraient suggérer une nouvelle alternative à cette question, l'existence d'espaces publics plurifonctionnels complexes. thomas.hutin01@gmail.com

If the issue of built markets for the Mediterranean Antiquity is a recurring question, the situation is, in many ways, different for the Celtic world. For a long time, the reflections about selling spaces were indeed pushed into the background of scientific publications. The last three decades, the excavations realized on several major oppida and other open settlements have gradually revealed the complexity of large urban centers for the Second Iron Age. It is in this that archaeological excavations on the Corent's hillfort helped to uncover a vast complex open onto inside courtyard. Its artisanal and commercial vocations are not to be reconsidered. Structured and optimized for the sale of various goods, this urban structure perfectly matches the image that we can have of architectural constructions. Despite the growing amount of archaeological records, not one representative analogy was discovered by excavations. Some similar complexes have been identified in the western and central-eastern France, but none whose nature and function could be as significant. On the other hand, recent discoveries of large squares might suggest a new alternative to the research: the existence of complex multifunctional public spaces.

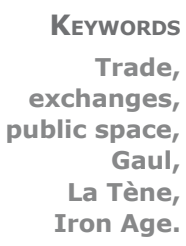


«Il ne peut pas y avoir de société qui ne sente le besoin d'entretenir et de raffermir, à intervalles réguliers, les sentiments collectifs et les idées collectives qui font son unité et sa personnalité. Or, cette réfection morale ne peut être obtenue qu'au moyen de réunions, d'assemblées, de congrégations où les individus, étroitement rapprochés les uns des autres, réaffirment en commun leurs communs sentiments

[...] » Durkheim 1960 [1912], p. 610.

Si pendant longtemps les querelles incessantes entre primitivistes et modernistes ont grandement modelé notre conception de I'histoire économique, le recours à la donnée archéologique permet aujourd'hui de porter un regard neuf sur ce pan de notre histoire. De la même manière qu'a pu l'initier le colloque interdisciplinaire d'Athènes Tout vendre tout acheter. Structures et équipements des marchés antiques, cette contribution se veut comme un essai de restitution « du cadre concret des échanges $\gg[1]$.

Éléments essentiels des centres urbains actuels, les espaces de vente (échoppes, boutiques, places marchandes, etc.) font de nos jours partie intégrante de l'armature et de la composition urbaines de nos villes. Si leurs identifications ne posent pas véritablement problème aujourd'hui, leur reconnaissance sur le terrain semble moins aisée pour la période celtique. Souvent jugés - et à raison - comme diffus [2] et difficilement interprétables, ces derniers ne font qu'à de très rares occasions I'objet d'une étude approfondie.

Pour reprendre la définition donnée par Ch. Blein, ces espaces de vente désignent dans le cas présent les « différentes constructions implantées au cœur des villes et dans lesquelles prenait place la vente de marchandises variées » [3]. Cette définition correspond en grande partie au deuxième sens que donnent les urbanistes du «magasin »: comme le lieu où prend place la vente de produits plus ou moins spécialisés et à destination d'une clientèle ciblée [4].

Cette recherche de vestiges d'aménagements spécialisés et optimisés aux pratiques de l'échange sera envisagée entre le $\mathrm{III}^{\mathrm{e}}$ et le $\mathrm{I}^{\mathrm{er}} \mathrm{s}$. av. J.-C en Gaule. Cette échelle de temps correspond aux profonds changements socio-économiques qui s'opèrent dans la société gauloise dans la deuxième moitié de la période laténienne.

[1] Si une partie des informations sont issues d'un travail de master soutenu en 2014 à l'université de Bordeaux Montaigne, voir Hutin 2014, I'objectif de cette contribution est essentiellement similaire aux problématiques et réflexions envisagées par le colloque d'Athènes qui s'est tenu en 2009, voir CHANKOWSKI \& KARVONIS 2012, p. 13.
Parmi ces nouveautés, I'utilisation de la monnaie dans les échanges marchands est le signe indéniable d'un nouveau langage économique. En très peu de temps, elle passe d'un statut de bien de prestige à celui d'équivalent général des biens. Du point de vue de I'occupation du territoire, le paysage majoritairement composé d'habitats isolés évolue peu à peu et voit l'apparition de toutes nouvelles formes d'habitats groupés aux caractéristiques originales. Villages agricoles (ex : AcyRomance), agglomérations artisanales (ex : Lacoste) à La Tène $\mathrm{C} 1-\mathrm{C} 2$ et oppida pour La Tène D1-D2 viennent en effet se greffer à ce paysage fondamentalement rural [5]. Sauf exception, le choix de ces centres urbains comme objet d'étude a grandement été orienté par le fait qu'ils incarnent des situations propices aux pratiques de l'échange marchand, et par conséquent semblent favorables à l'identification de structures dédiées à ces activités. Ainsi, les oppida de Corent (Puy-deDôme), du Titelberg (Grand-Duché de Luxembourg), de Villeneuve-Saint-Germain (Aisne), le village agricole d'Acy-Romance (Ardennes) constitueront les principaux exemples sur lesquels s'appuie cette étude.

\section{ANALYSE ARCHITECTURALE ET FONCTIONNELLE DU « COMPLEXE C» DE L'OPPIDUM DE CORENT (PUY-DE-DÔME)}

Parmi les oppida les plus intensément fouillés ces dix dernières années figure le plateau basaltique du «Puy de Corent ». Conditionnée par ses origines volcaniques, cette immense forteresse naturelle de 70 ha présente une configuration sur deux niveaux topographiques : une partie haute, correspondant au cône éruptif à l'origine du plateau, et une partie basse en pente douce formée par l'épanchement des laves massives.

[2] FICHTL 2005, p. 139.

[3] BLEIN 2012, p. 163.

[4] MerLin \& ChOAY 2005, p. 511.

[5] FICHTL 2013, p. 21 ; BuChSENSCHUTZ 2008, p. 253. 


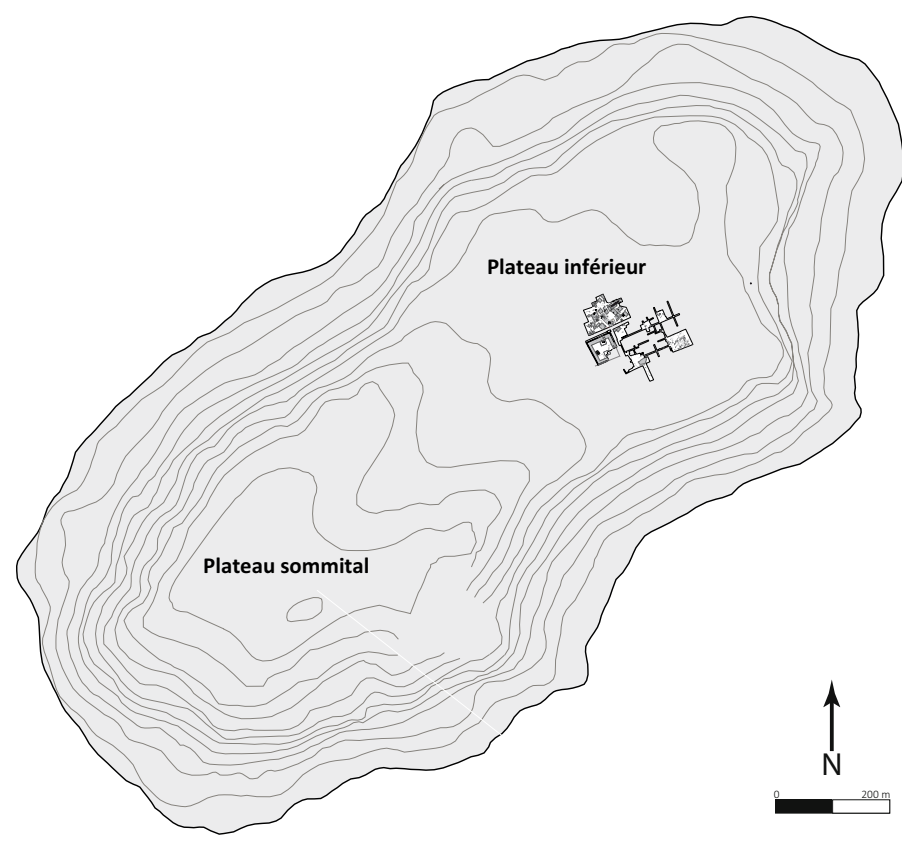

A Figure 1 : plan général du plateau du « Puy de Corent » (d'après DemierRe \& Poux 2012, p. 210).

$\checkmark$ Figure 2 : plan général des infrastructures publiques de l'oppidum de Corent (FICHTL 2012, p. 48).

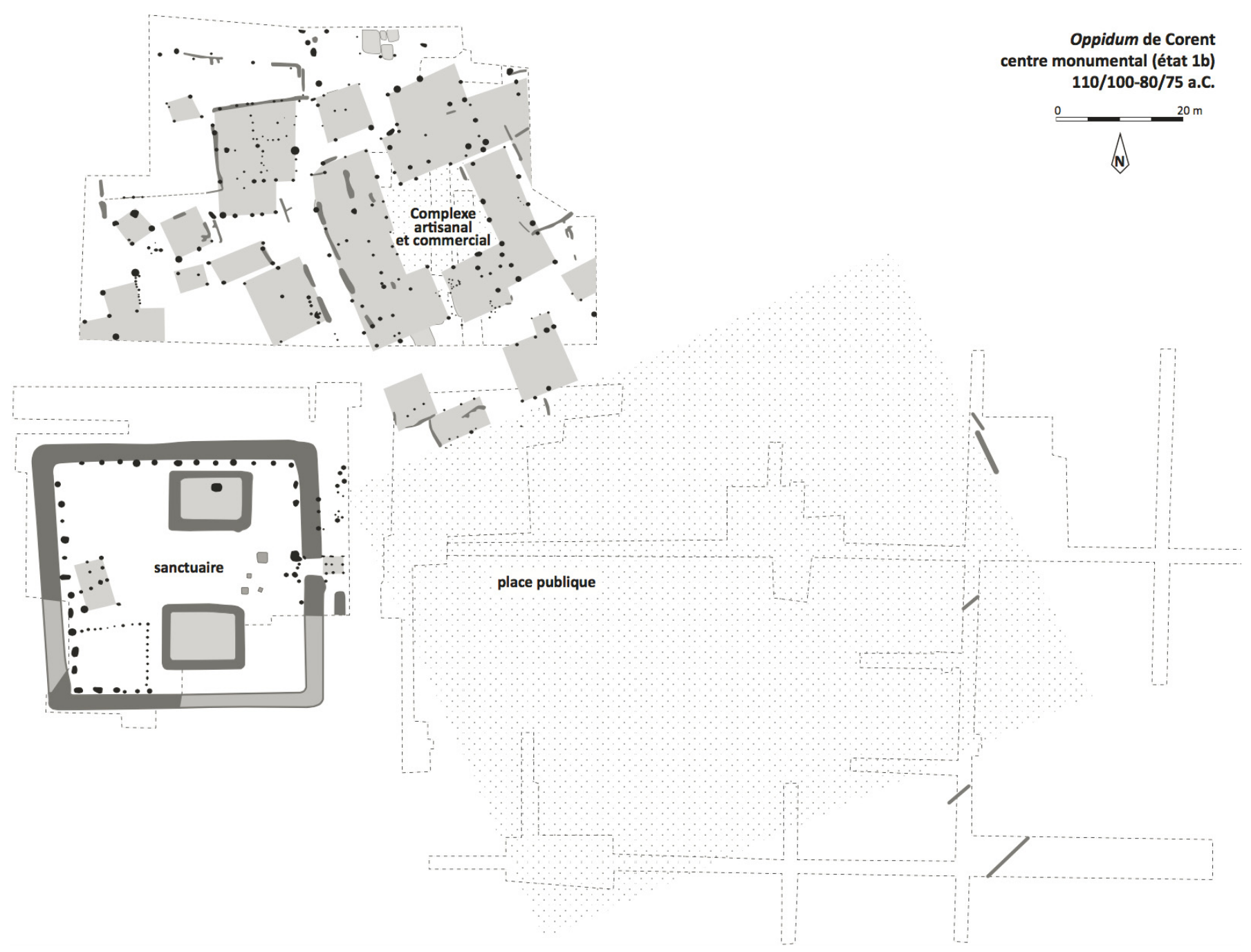

C'est à ce titre que les opérations archéologiques entreprises depuis 2001 sous la direction de M. Poux ont d'une communauté de milliers d'habitants. En peu de temps, l'oppidum se pare d'un système urbain cohérent principalement illustré par un réseau viaire complexe, des édifices communautaires et collectifs ainsi que de nombreux îlots d'habitations (fig. 1).

Situé au nord-est du sanctuaire monumental et au nord de l'esplanade, le « complexe $C$ » (fig. 2) prend la forme d'un vaste complexe architectural sur cour à vocation commerciale et artisanale [6]. Son édification est datée des années 130-120 av. J.-C. et sa désaffection aux environs de 50 av. J.-C. Dès son origine, cet ensemble s'organise en sept bâtiments discontinus alignés sur un axe nord-sud et circonscrivant un espace central à ciel ouvert de $352 \mathrm{~m}^{2}$ (fig. 3). Si son niveau de sol, homogène et aplani, ne présente aucune structure porteuse dans sa partie interne, en bordure plusieurs aménagements pourraient correspondre à des portiques ou des appentis. Les

[6] Poux 2006, 2007 et 2008. permis de mettre en évidence au niveau inférieur plusieurs séries d'aménagements répondant aux besoins 


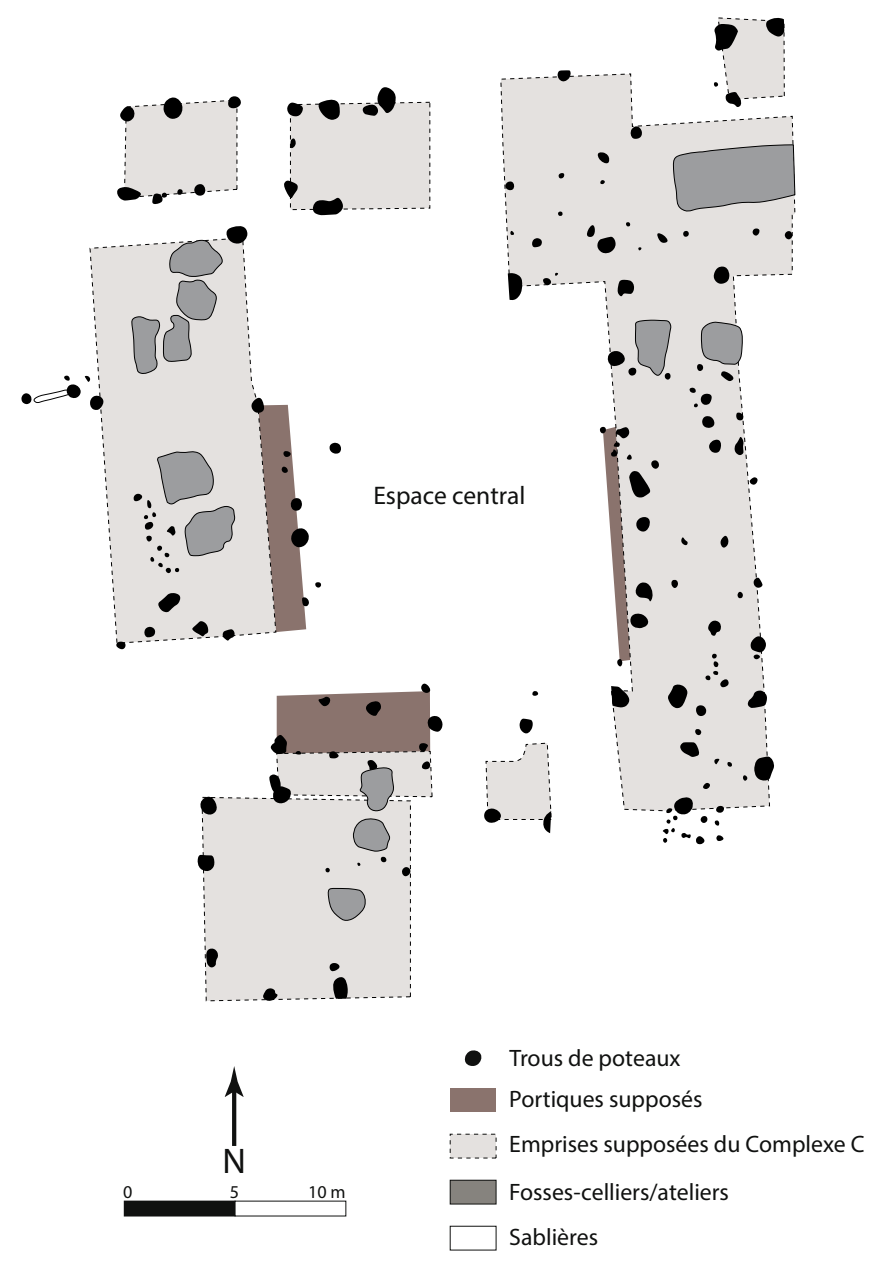

Figure 3

État la du complexe C (d'après Poux 2007, p. 63).

accès situés dans les parties méridionale et occidentale sont au nombre de trois et permettent de communiquer librement avec les quartiers et les espaces de voirie environnants. Si la morphologie de cet ensemble ne semble guère évoluer pendant plusieurs dizaines d'années, les remaniements urbanistiques de l'oppidum, à la transition de La Tène D1-D2, vont avoir comme principale conséquence une réorientation des bâtiments en direction du nord-ouest et une transformation radicale de sa structure. Son plan $(40 \times 30 \mathrm{~m})$ se cristallise alors autour de quatre branches de bâtiments contigus toujours disposés autour d'une cour centrale de $300 \mathrm{~m}^{2}$ (fig. 4). Par la suite, peu de changements sont à signaler hormis le passage de structures en matériaux périssables à une architecture empierrée.

La vocation commerciale trouve sa justification dans les monnaies en bronze et en argent (fig. 5.1) pour la plupart associées avec de nombreux jetons en céramique (fig. 5.2). Plusieurs centaines de jetons ont également été découvertes au niveau de la zone d'entrée et

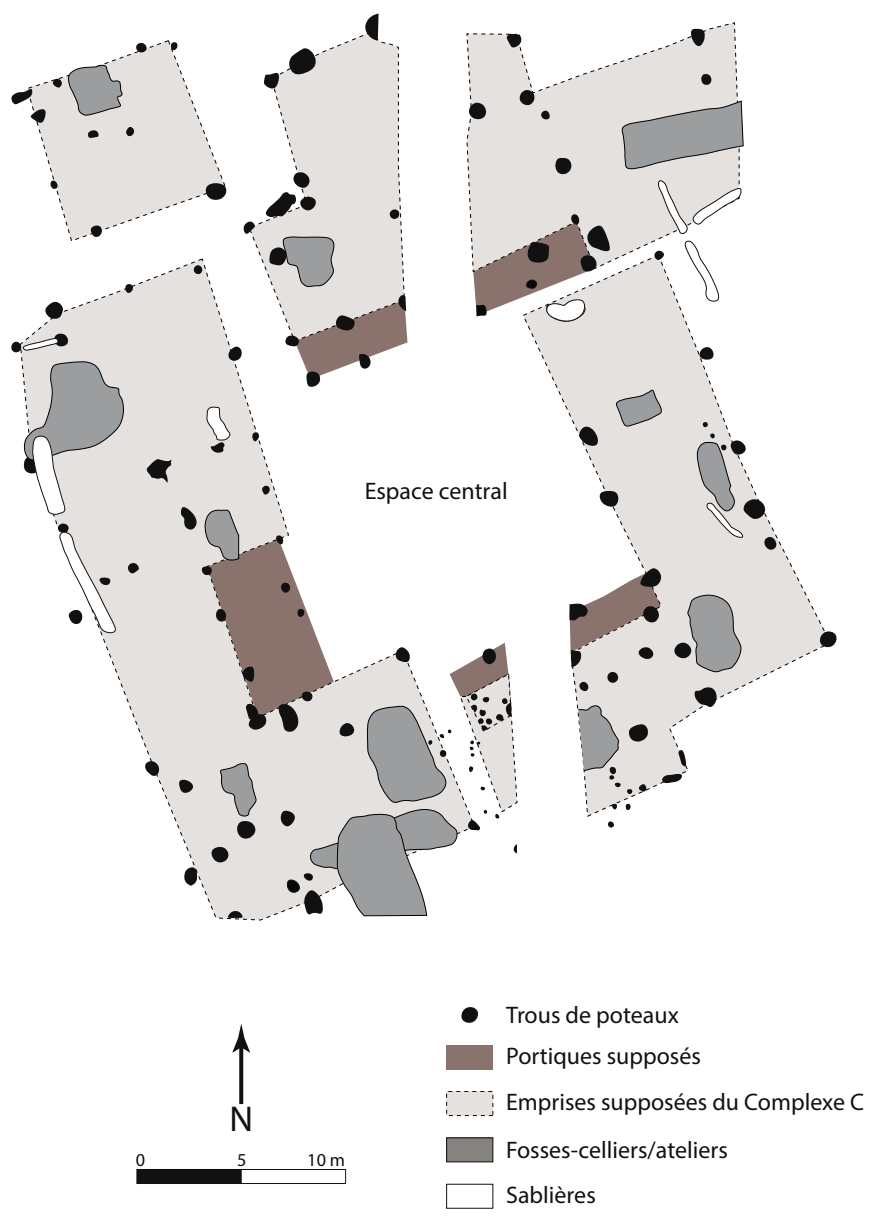

Figure 4

État Ib du complexe C (d'après Poux 2007, p. 64).

des enclos intérieurs du sanctuaire, mais il semble peu probable que les significations fonctionnelles soient les mêmes que pour le complexe $C$. Le contexte de découverte revêt ici un rôle particulièrement important dans I'attribution fonctionnelle de ce petit mobilier. En effet, le contexte cultuel et communautaire du sanctuaire associé à l'esplanade tendrait à interpréter les jetons comme des offrandes, ou alors comme les témoins éventuels de pratiques de comptabilité, de vote ou de libéralités [7]. A contrario, le caractère essentiellement profane que dégage le complexe $C$ supposerait un usage principalement lié aux opérations artisanales, d'échanges marchands et de comptage qui se déroulaient à l'intérieur de cet aménagement. Ce petit mobilier réparti surtout dans les angles de la cour, sous de possibles portiques, et dans une moindre mesure au centre de la cour constituerait ici un marqueur d'activités liées aux échanges marchands [8]. À cela il faut ajouter la découverte associée d'éléments de pesée comme des fléaux de balances et des poids en plomb renforçant encore cette attribution. 
$\checkmark$ Figure 5. 1

Carte de répartition des monnaies

(d'après DemierRe \& Poux 2012, p. 222).

$$
\begin{aligned}
& \text { Habitat (G) } \frac{0}{0000} \\
& \text { \% द वरदिo }
\end{aligned}
$$

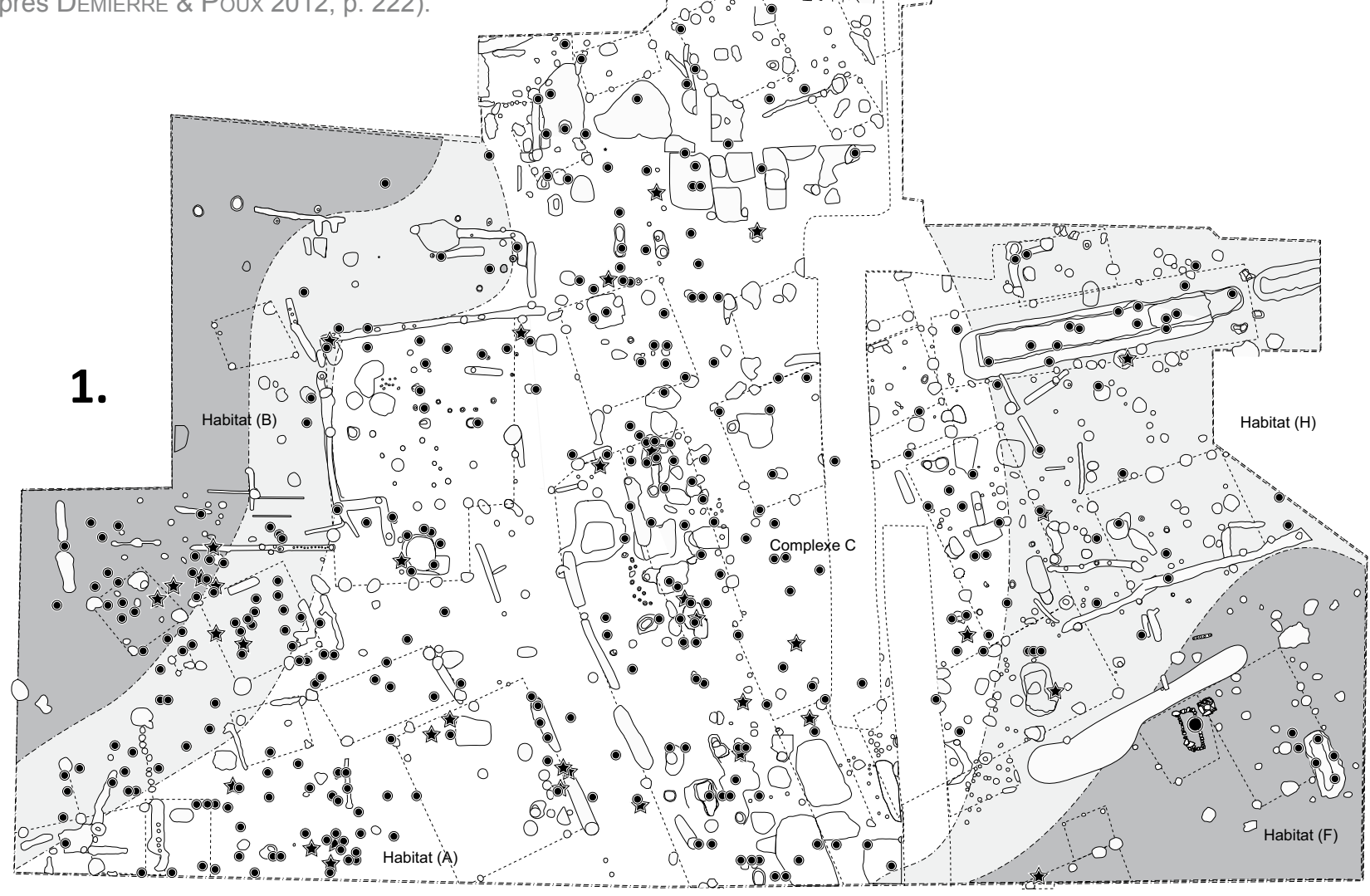

$\checkmark$ Figure 5.2

Carte de répartition des jetons en céramique

(d'après DemierRE \& Poux 2012, p. 222)
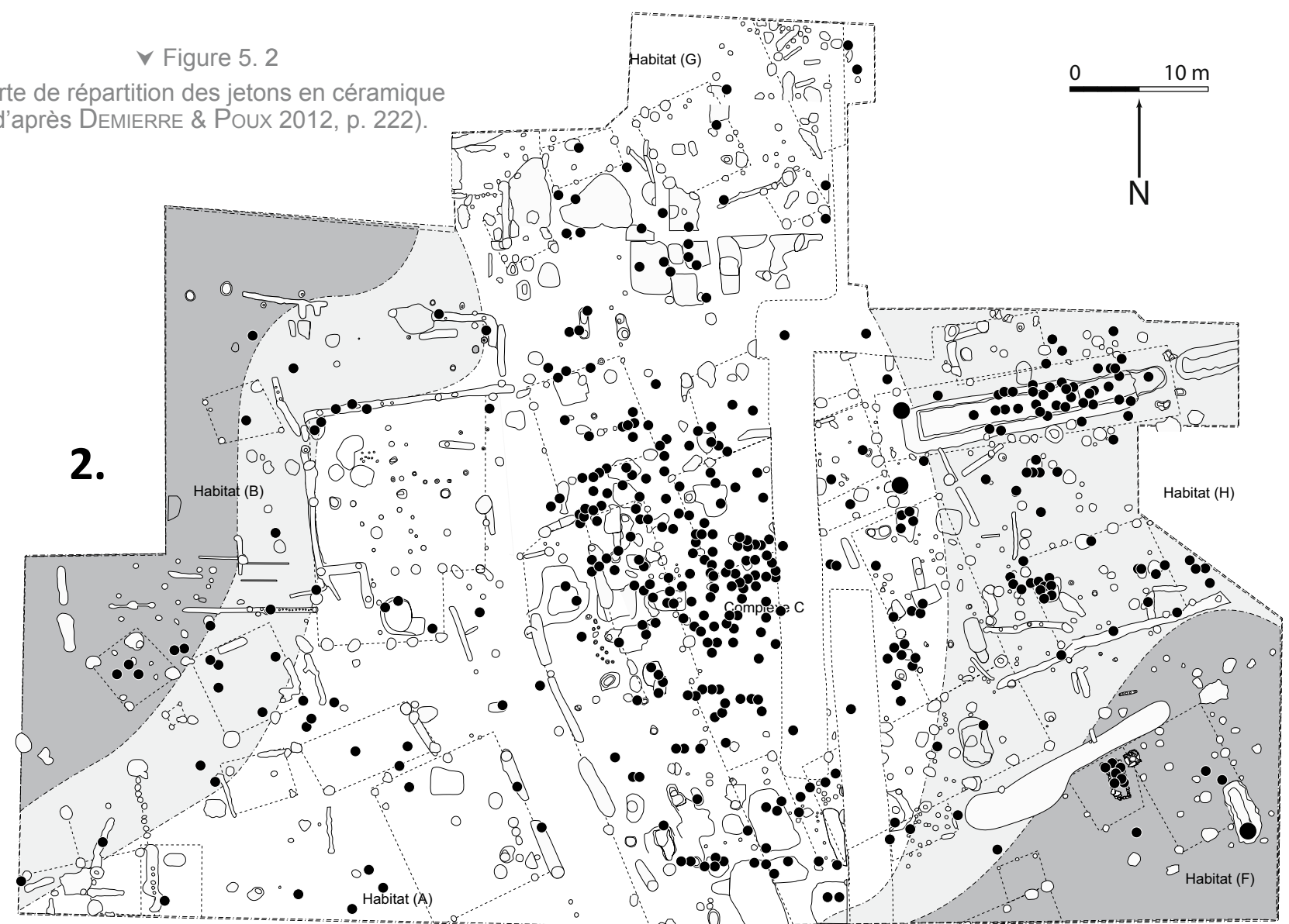
Dans cette même perspective, nous mentionnerons la présence dans l'angle nord-est d'un vaste bâtiment à trois nefs de $150 \mathrm{~m}^{2}$ abritant une cave d'une capacité de $80 \mathrm{~m}^{3}$. La fouille de son remplissage a révélé la présence d'une vingtaine d'amphores vinaires, plus ou moins complètes, et de nombreux tessons (plus de $5 \mathrm{t}$ ) empilés volontairement au fond du creusement. Ses abords ont eux aussi livré de nombreux éléments de mobilier : fragments de situles, de cruches, de passoires, de gobelets, de céramiques, de verre, de jetons, de monnaies ou encore de cols d'amphores sabrées. Tous ces éléments participent à l'image d'un bâtiment de type halle dont les fonctions principales devaient être le stockage, la consommation et la transaction de vin à hauteur de plusieurs milliers d'hectolitres.

Dans le monde classique, il est traditionnellement acquis que la vente de marchandises peut être réalisée parallèlement à d'autres types d'activités comme la production et le stockage [9]. À Pella, I'agora située au cœur de la ville occupe une superficie de près de 7,2 ha $(261,70 x$ $238 \mathrm{~m}$ ). De type « ionien », par opposition aux agorai dites «primitives », I'agora de Pella - au même titre que l'agora sud de Milet - se définit comme un bloc unitaire quadrangulaire intégré au reste du tissu urbain [10] (fig. 6). Si les portiques nord et I'angle sud-ouest semblent dévolus à des fonctions civiques et religieuses, les portiques sud et ouest étaient principalement destinés aux activités commerciales et artisanales (ateliers de

Figure 6 : plan de l'agora de Pella (GINOuvĖs 1993, p. 95).

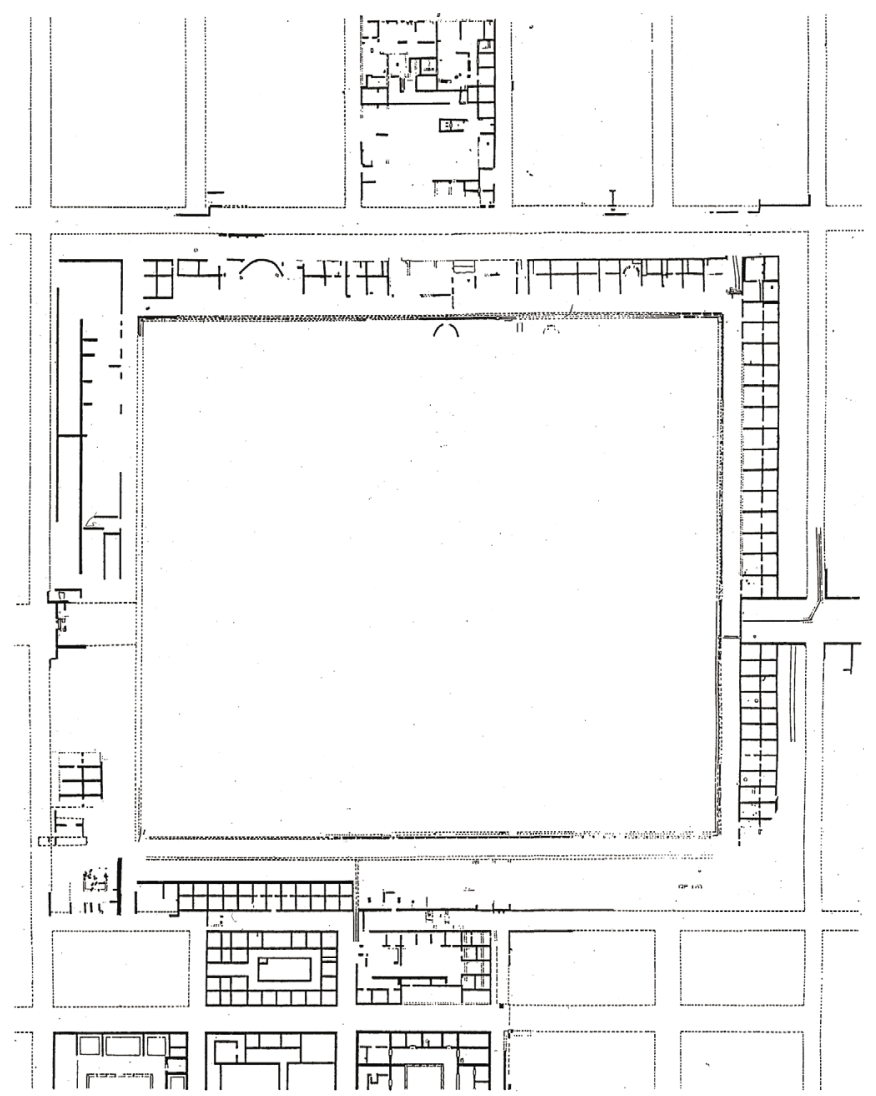

potiers, de coroplathes, travail des métaux, ainsi que des espaces de vente et d'exposition) [11]. Cette observation peut aussi être vérifiée pour le complexe monumental de Corent. Si les activités marchandes, et plus largement économiques (jetons en céramique, monnaies, éléments de pesée, etc.) sont principalement attestées en bordure de la place centrale, les activités de production et de transformation semblent se répartir au sein des cellules occidentales du complexe. Dans cet espace ont en effet été identifiées plusieurs structures à combustion, plaques foyères et foyers, et cavités interprétées comme des ateliers semi-excavés. Le comblement de certaines de ces fosses-ateliers a livré des marqueurs artisanaux en grande quantité : fragments de creusets, de moules et de lingotières associés à des outils lithiques et métallurgiques, des semi-produits, etc [12].

\section{UN UNICUM EN CONTEXTE CELTIQUE ?}

Si aucun doute ne subsiste quant à la vocation artisanale et commerciale du complexe $C$, son statut d'unique exemplaire en Gaule, et plus largement en contexte celtique, pose quelque peu problème. Sa qualité d'unicum peut toutefois être remise en question par de récentes découvertes archéologiques dans l'ouest et le centre-est de la France.

À Trégueux en Côte d'Armor [13], la fouille réalisée avant la construction d'une rocade a permis de mettre en évidence un bâtiment avec cour quadrangulaire de $36 \mathrm{~m}$ de côté daté de La Tène finale (fig. 7). Dans sa première phase, le bâtiment dont l'accès se trouve à l'est [14] est constitué de quatre galeries périphériques tournées vers une cour intérieure de $625 \mathrm{~m}^{2}$. L'état suivant est sensiblement le même à l'exception d'une rangée supplémentaire de poteaux pour chacun des côtés. Les seuls aménagements identifiés au centre de la cour sont quatre trous de poteaux dont les pendants à l'ouest ont été détruits par deux fossés d'époque moderne. Il est possible qu'à l'origine un édifice hexagonal ait occupé le centre de cette cour [15]. Il faut enfin ajouter à cela un enclos fossoyé constitué de deux entrées à bords rentrants, à l'est et à l'ouest,

[9] BLEIN 2012, p. 163.

[10] Ibid., p. 164.

[11] Ibid., p. 164-165. ; GINOUVĖs 1993, p. 91-97.

[12] Pour de plus amples informations au sujet de la répartition du mobilier et des structures, voir DemieRre \& Poux 2012.

[13] AlLen et al. 2012.

[14] Ibid., p. 177.

[15] Ibid., p. 175. 


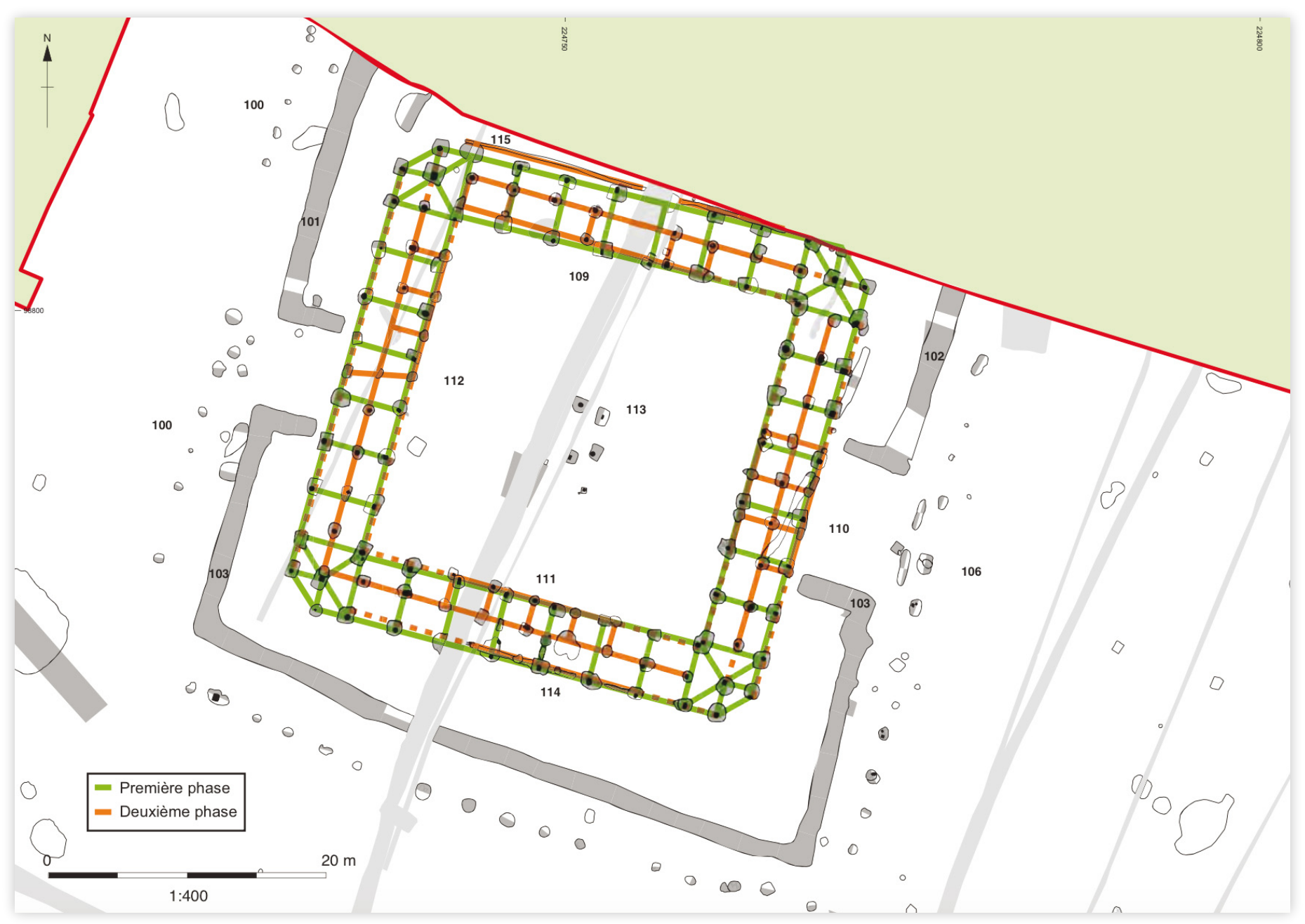

A Figure 7 : plan du bâtiment sur cour du site de Trégueux en Bretagne (AlLEN et al. 2012, p. 669).

entourant l'ensemble du bâtiment avec cour. La relation qu'il a pu exister entre ces deux infrastructures reste toutefois incertaine.

Ce type de bâtiment a également été observé au Mont-Beuvray, dans le secteur du Parc aux Chevaux. C'est un grand complexe monumental bordé par deux voies, l'une à l'ouest et l'autre au sud [16] (fig. 8). Daté du milieu du I ${ }^{\text {er }}$ s. av. J.-C., cet enclos quadrilatère de $43 \mathrm{~m}$ de long était formé d'au moins trois galeries périphériques tournées, comme à Trégueux, en direction d'un espace central de $20 \mathrm{~m}$ de côté. Si les réaménagements successifs gardent les caractéristiques architecturales du premier état, c'est au cours de la troisième et dernière phase que les changements sont les plus remarquables. D'une part, les galeries doubles puis simples des états précédents sont entièrement restructurées par la mise en place d'une palissade monumentale, d'autre part, la cour interne jusqu'alors vide de construction se voit dotée d'un grand bâtiment partiellement reconnu [17].

[16] Nouvel \& Barral 2014, p. 93-145.

[17] Ibid., p. 111 et 117.
Il est difficile de s'exprimer quant à la qualité fonctionnelle de ces aménagements même si deux hypothèses peuvent retenir notre attention : La première consiste par analogie avec le macellum romain en une place de

$\checkmark$ Figure 8 : restitution en plan du complexe monumental sur cour de Bibracte (PC15) dans son état II (NouvEL \& BARRAL 2014, p. 113)

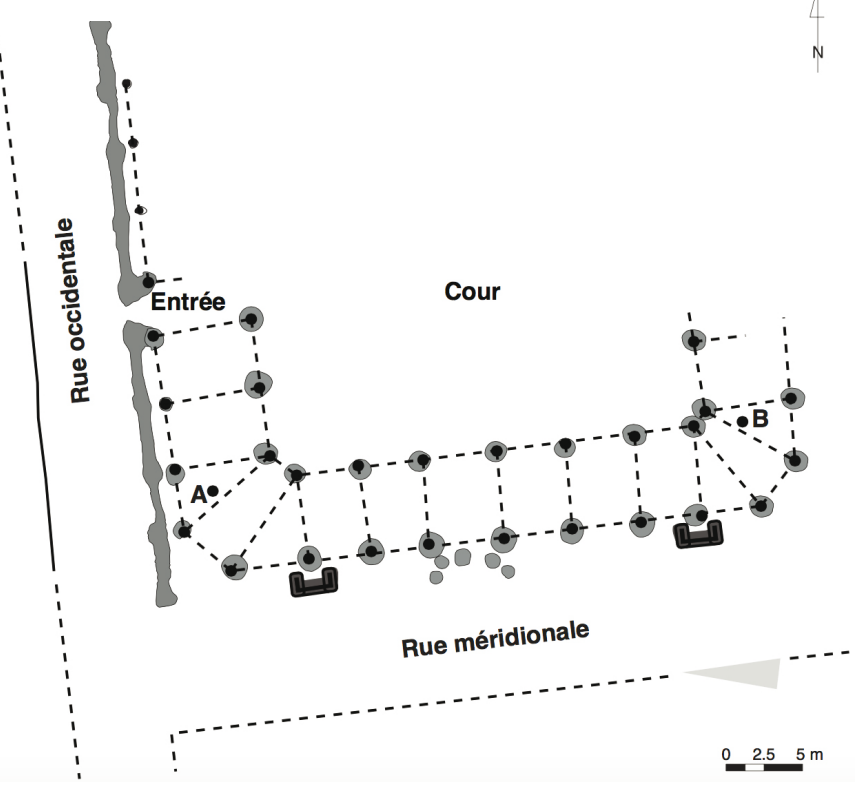


marché formant un bloc unitaire, centrée sur une cour interne [18]. La seconde en un lieu de culte à l'image du plan du sanctuaire de Corent [19]. Malgré tout, dans l'une comme dans l'autre, les fouilleurs insistent sur l'absence d'assemblage de mobiliers caractéristiques pouvant évoquer ce type d'activités.

Si un parallèle architectural peut être éventuellement suggéré avec les édifices des Natteries à Cholet (Maine-et-Loire) [20] ou de Paule au « Camp de SaintSymphorien » (Côte d'Armor) [21], la comparaison se retrouve bien vite limitée en raison d'une attribution fonctionnelle bien différente (écurie ou stabulation) et d'un contexte de découverte bien distinct. D'un côté les sites de Cholet et de Paule sont de véritables habitats aristocratiques, tandis que d'un autre Bibracte et Trégueux se présentent I'un comme un oppidum, l'autre comme une agglomération ouverte.

\section{VERS LA DÉFINITION D'ESPACES RÉSERVÉS AUX USAGES COLLECTIFS ET COMMUNAUTAIRES DANS LES CENTRES URBAINS DE LA TĖNE FINALE EN GAULE}

Quel que soit le crédit alloué à ces analogies, aucune n'est directement comparable, dans l'état, à l'ensemble monumental de Corent. Dès lors, ne s'agit-il pas d'un problème $d$ 'identification et ne faudrait-il pas rechercher d'autres formes de structures, ou d'espaces, liées aux activités non plus seulement économiques et commerciales ? C'est à ce titre que plusieurs exemples permettent d'aborder cette question.

\section{RETOUR À CORENT}

Les fouilles effectuées au centre de l'oppidum depuis les années 2000 ont permis de mettre en évidence un sanctuaire monumental fondé dès la seconde moitié du $\mathrm{II}^{\mathrm{e}} \mathrm{s}$. av. J.-C. Son seul accès prenait la forme d'une tour-porche s'ouvrant sur une grande esplanade vide de construction. Elle se présente sous la forme d'une aire ouverte de type « parvis » d'environ $4000 \mathrm{~m}^{2}$, plus ou moins orientée sur un axe est-ouest. Les accès sont rendus possibles sur ses côtés nord, sud et est par le biais de plusieurs voies de communication. Le remaniement que subit I'oppidum

[18] Allen et al. 2012, p. 478.

[19] Nouvel \& BarRal 2014, p. 143.

[20] MAGUER 2007.

[21] MENEZ 2009.

[22] Metzler 1991, p. 28-29; Metzler et al. 2006, p. 203-204.

[23] MetZler 2008, p. 157. à la transition La Tène D1-D2 affecte l'esplanade : sa forme devient plus irrégulière, ses limites sont altérées et le changement d'orientation des îlots adjacents produit une extension de sa surface au sol de plus de $1500 \mathrm{~m}^{2}$. Si la rareté du mobilier recueilli n'offre que peu d'indices quant aux activités qui devaient y être pratiquées, la situation dans le prolongement exact du sanctuaire et à quelques pas du complexe artisanal et commercial doit être considérée comme un premier élément de réponse. En envisageant une certaine filiation fonctionnelle entre ces trois aménagements, cette esplanade a bien pu accueillir à la fois des manifestations d'ordre cérémoniel, économique et même politico-juridique.

\section{L'OPPIDUM DU TITELBERG}

Avec ce type d'esplanade, l'immense centre public du Titelberg reste sûrement l'exemple le plus représentatif de cet exemple d'aménagement. C'est en effet sur le principal oppidum des Trévires qu'a été mise en évidence une zone de 10 ha pour ainsi dire vide de construction et principalement délimitée par un fossé monumental d'environ $5 \mathrm{~m}$ de large pour 2,50 $\mathrm{m}$ de profondeur, doublé d'un mur de briques crues (fig. 9). D'abord suggérée par photographie aérienne [22], la zone enclose prend la forme d'un espace polygonal, strictement isolé du reste de I'habitat, dont le seul secteur aménagé se situe au carrefour de la voie principale et du fossé de clôture. Le premier aménagement daté de la fin du $\mathrm{II}^{\mathrm{e}} \mathrm{s}$. av. J.-C. se présente comme quatre palissades formant trois travées de plus de $60 \mathrm{~m}$ de long interprétées comme un dispositif amovible lié à la tenue d'assemblées politiques [23] (fig. 10.1). Un aménagement similaire a été mis en évidence dans le sanctuaire de Gournay-sur-Aronde.

Figure 9 : plan général de l'oppidum du Titelberg (d'après FICHTL 2012, p. 45).






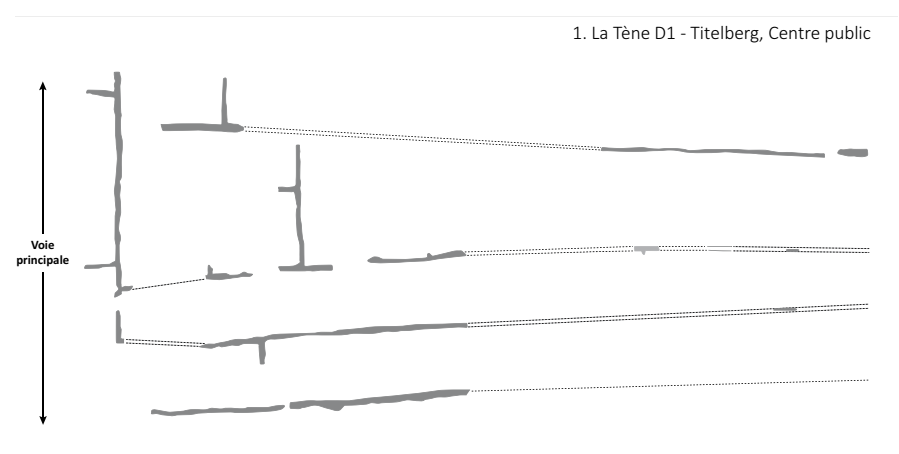

Figure 10.1

Aménagements palissadés identifiés pour La Tène D1 (d'après METZLER 2008, p. 158).

Il s'agit là encore de quatre palissades parallèles, formant des travées d'environ 2,70 m de large.

Dans le courant du $\mathrm{I}^{\mathrm{er}}$ s. av. J.-C., cette structure est rapidement démantelée pour laisser place à une grande halle monumentale de $210 \mathrm{~m}^{2}$ (fig. 10.2). Si une unique fonction religieuse ne peut être tout à fait exclue pour cet édifice, les milliers d'ossements retrouvés dans son périmètre renvoient plutôt l'image de rassemblements à caractères économiques [24]. Les quarante mètres qui la séparent de la voie principale sont constitués d'une esplanade uniquement occupée par une fondation quadrangulaire interprétée comme un autel [25] et bordée par plusieurs fosses et foyers ainsi qu'une structure longitudinale dont la nature reste à préciser.

Quelle fonction conférer à cette immense aire ouverte ? Le premier élément de réponse pourrait se trouver dans un passage du Bellum Gallicum : « C'est là, selon I'usage des Gaulois, le commencement de la guerre : une loi commune oblige tous ceux qui ont l'âge d'homme à y venir en armes ; celui qui arrive le dernier est mis à mort, sous les yeux de la multitude, dans de cruels supplices » (César, BG, V, LVI). Même si César ne précise pas si des rassemblements de ce type se sont produits dans I'oppidum lui-même, la dizaine d'hectares permet de l'envisager. De la même façon, la diversité et l'importance des rejets osseux suggèrent la pratique d'une grande variété d'activités, propres à tout champ de foire [26].

\section{L'OPPIDUM DE VILLENEUVE-SAINT-GERMAIN}

Repérés dès 1976 par prospections aériennes et fouillés jusqu'à encore récemment [27], les quatre fossés de I'oppidum de Villeneuve-Saint-Germain restent à ce jour les seuls aménagements de ce type connus pour l'ensemble du monde celtique (fig. 11.1). D'une longueur totale estimée à $1250 \mathrm{~m}$, d'une largeur oscillant entre 1,10 et $1,40 \mathrm{~m}$ pour une profondeur moyenne d'environ 1,40 m, chaque tronçon venait à converger de façon longitudinale vers un étroit carrefour sans pour autant se
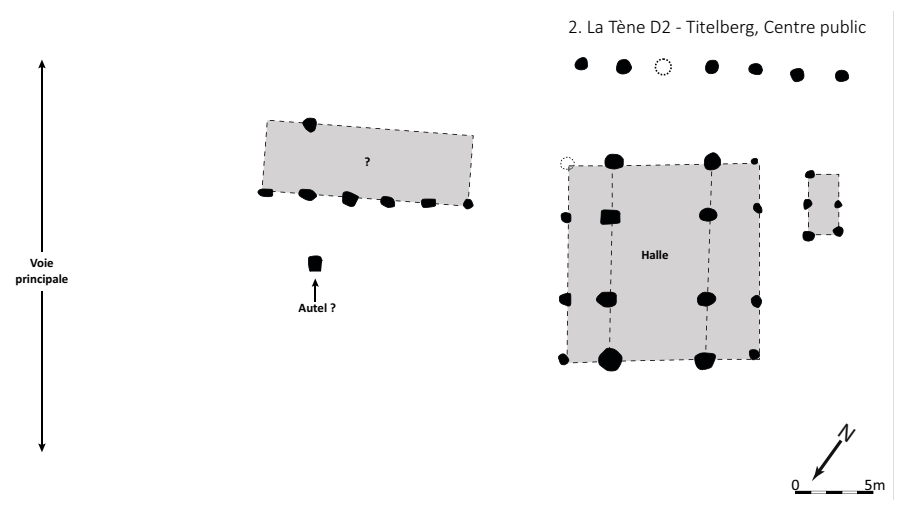

Figure 10.2

Aménagements identifiés pour La Tène $\mathrm{D} 2$, dont une grande halle monumentale à trois nefs (d'après METZLER 2008, p. 159).

couper. Tandis que les fonds des fossés présentent des profils assez irréguliers, ondulés et sans grande déclivité, l'intérieur est aménagé de poteaux internes latéraux allant systématiquement par paires et creusés à intervalles réguliers dans les parois. En ce qui concerne leurs aménagements externes, ils présentent sur chacun de leurs côtés des rangées de poteaux régulièrement espacées, parfaitement alignées et de mêmes orientations que celles des fossés. Deux interprétations bien différentes peuvent aujourd'hui être retenues pour ce vaste aménagement. Il convient désormais de les présenter brièvement.

La première avancée en 1988 par J. Debord, B. Lambot et $O$. Buchsenschutz [28] supposerait une fonction essentiellement économique, celle de «galeries marchandes bordées d'étals »[29] (fig. 11.2). Dans cette hypothèse, les structures prennent toutes la forme d'une construction longiligne composée d'une nef centrale, recouvrant chaque fossé, et de deux coursives latérales. L'ensemble était possiblement abrité par une toiture à double pente dont la couverture se rejoignait à l'intersection des fossés orientaux, occidentaux et méridionaux tout en formant une entrée monumentale en son centre. Pour ce qui est des fossés, ils devaient être recouverts d'un plancher facilitant la circulation dans la nef centrale et constituant ainsi un « vide sanitaire »[30]. Pour ce qui est de la fonction marchande, celle-ci a été déduite des activités artisanales qui devaient se dérouler aux abords,

[24] Ibid., p. 158.

[25] MetZler et al. 2006, p. 196.

[26] MÉNIEL 2008, p. 172.

[27] L'exploration des fossés est marquée par deux grandes périodes : une première entre 1979 et 1983 par les équipes de J. Debord et de I'URA 12 du CNRS, et une seconde entre 2007 et 2011 par P. Ruby, voir Ruby \& AuXIETTE 2011 ; RuBY \& AUXIETTE 2013.

[28] DeBORD et al. 1988.

[29] DeBORD 1990, p. 150.

[30] Ibid., p. 150. 
comme en témoignent d'une part certaines fosses localisées entre 15 et $40 \mathrm{~m}$ de distance dont l'orientation était la même que celle des fossés, et d'autre part par le mobilier retrouvé dans leur remplissage témoignant d'activités de ce type.

La seconde interprétation est a priori aux antipodes de la première: il s'agirait de structures de vote (fig. 11.3). Proposée en 2000 par Chr. Peyre, I'hypothèse ne rejette pas tout à fait la proposition précédente. Toutefois et selon l'auteur, les usages devaient être divers, certaines activités pouvaient être «permanentes »[31], et d'autres «temporaires et amplifiées par un afflux de fréquentation momentanée du site, à l'occasion de rassemblements périodiques du peuple de la cité »[32]. La conséquence d'une telle proposition remet en cause la restitution précédemment présumée, supprimant alors la couverture en chaume et le toit en double pente pour y ajouter des passerelles comme superstructures assurant la surveillance des « opérations de vote » [33]. En somme, un chemin de ronde qui permettrait de canaliser les votants lors des opérations électives. Opérations qui seraient illustrées selon Chr. Peyre à la fois par la découverte de rouelles et de perles de verre, spécifiquement au carrefour des fossés (fonctionnant

[31] PeYRe 2000, p. 165.

[32] Ibid., p. 165.

[33] Ibid., p. 171.

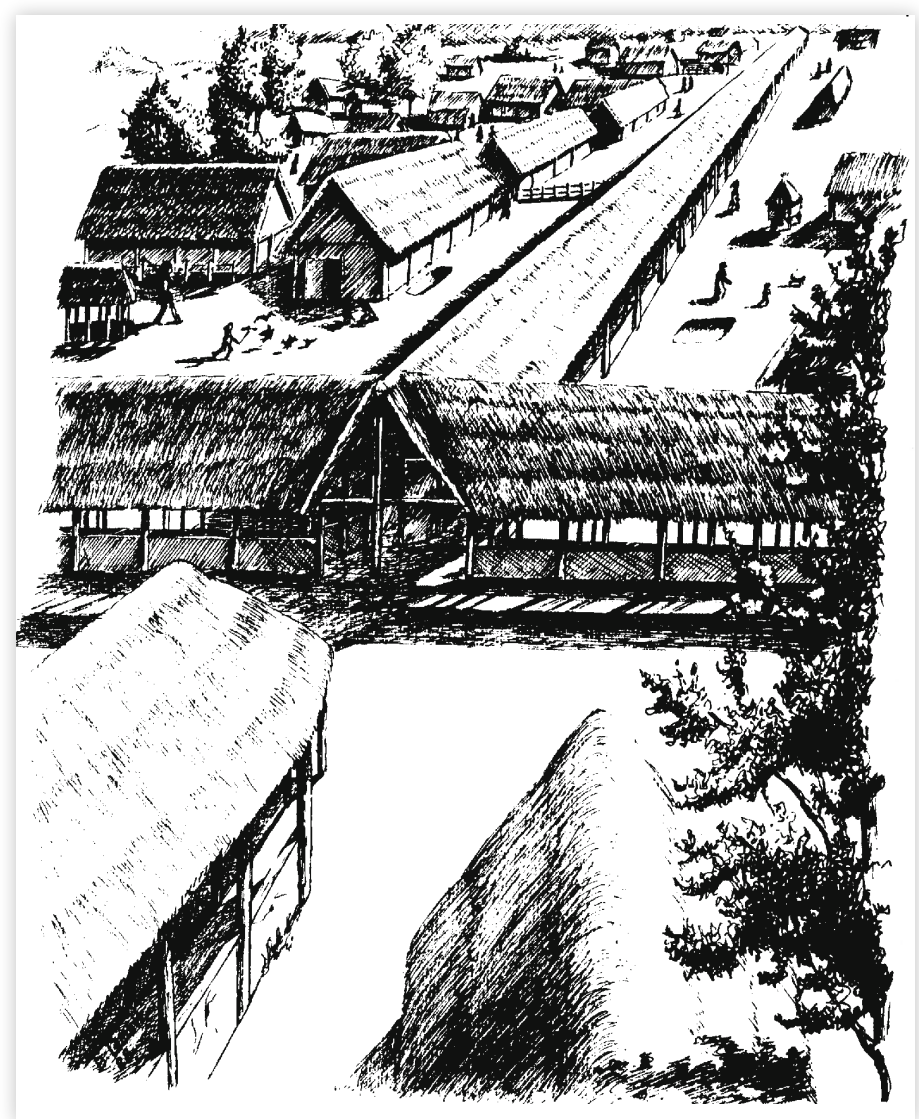

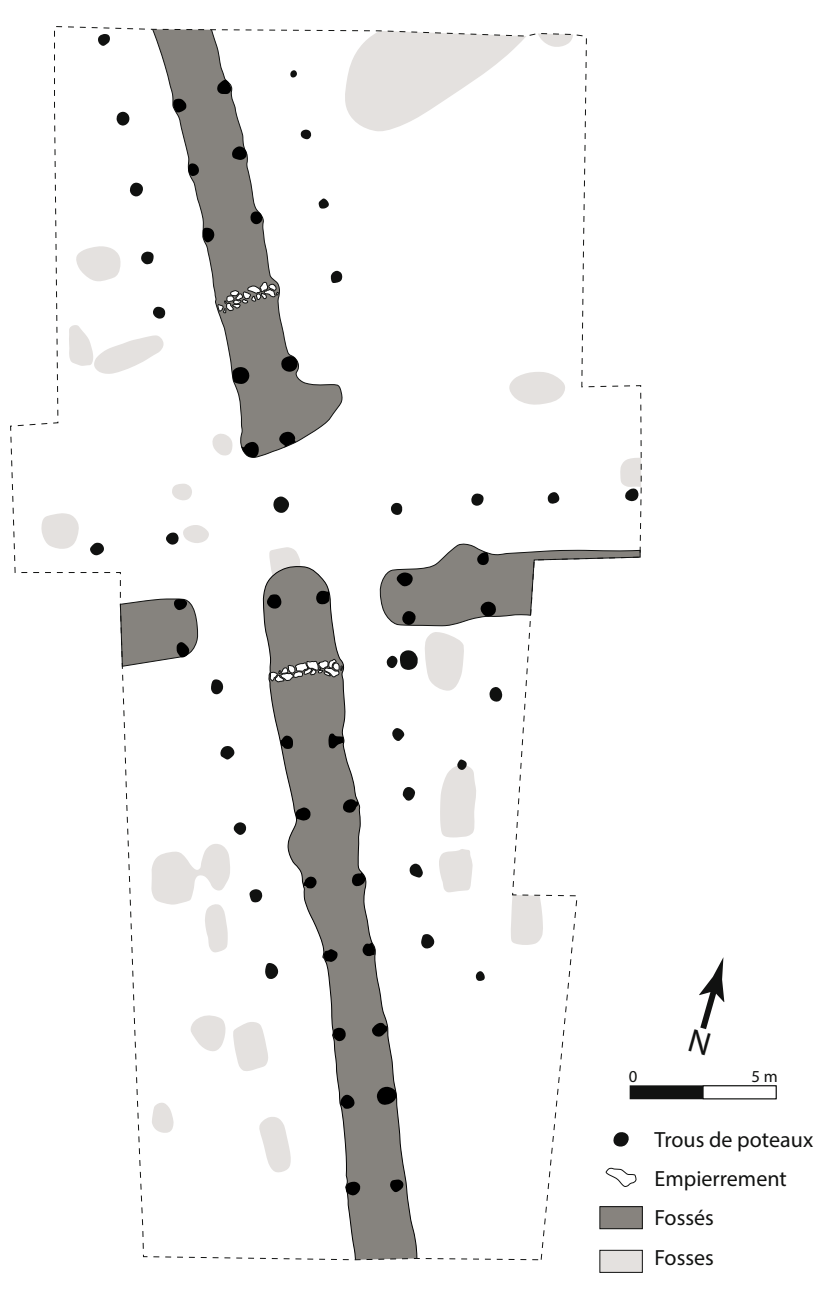

A Figure 11.1 : plan des fossés « en croix » au point d'intersection (d'après DEBORD et al. 1988, p. 121). $<$ Figure 11.2 : hypothèse de restitution des « galeries
marchandes » (DEBORD et al. 1988, p. 135).

$\checkmark$ Figure 11.3 : hypothèse de restitution des « installations de vote » (PEYRE 2000, p 171).
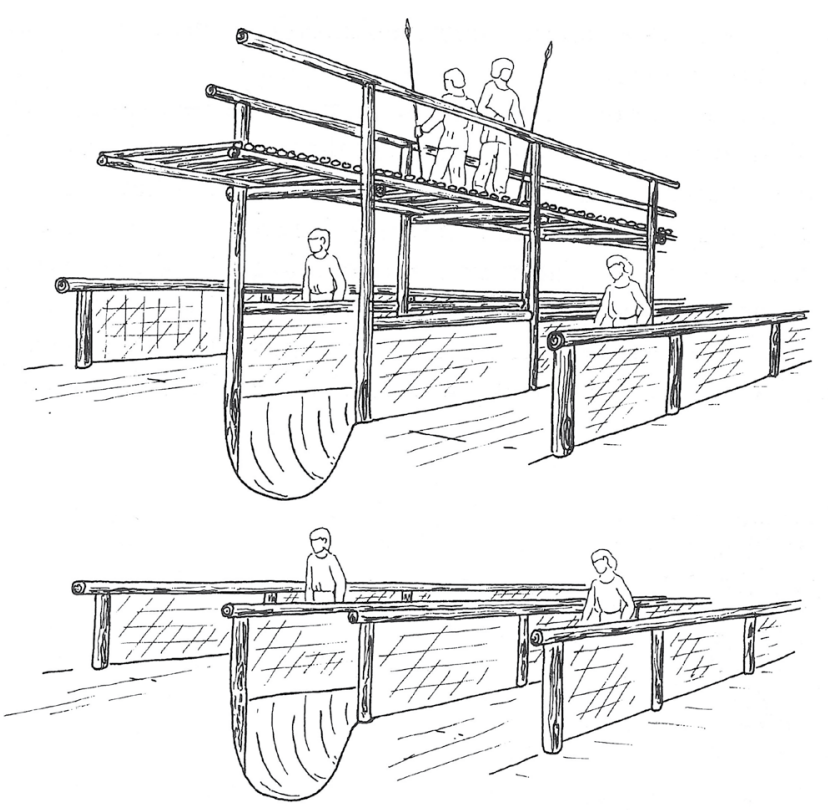


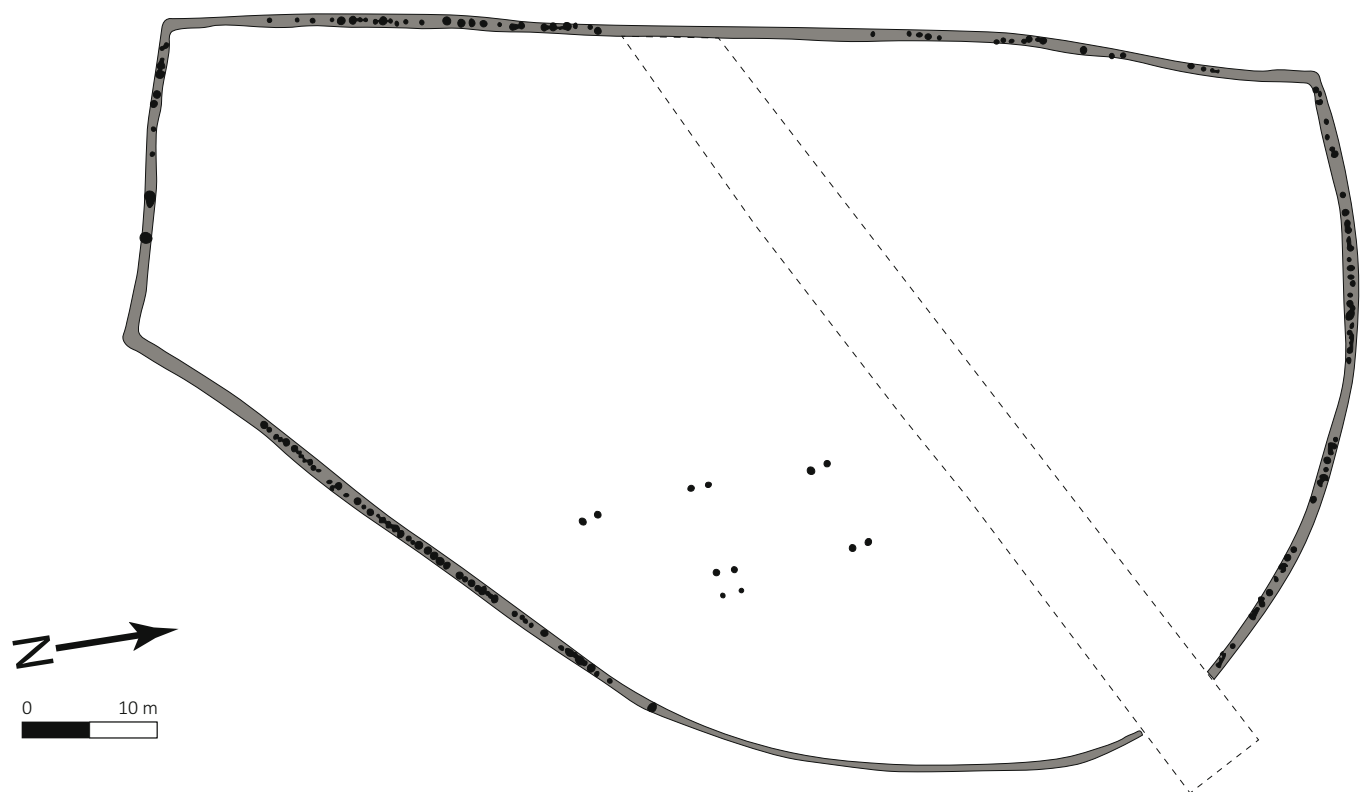

Figure 12

Place en « D » du village agricole d'Acy-Romance (d'après LAMBOT 2000, p. 27).

ainsi comme jetons de vote), mais aussi dans une perspective comparative avec les Saepta du Champ de Mars à Rome [34].

Si l'analogie est attirante, elle n'en reste pas moins risquée. Comme peut le rappeler $M$. Dondin-Payre, et à très juste titre, pour les installations du Titelberg : « La comparaison entre les installations d'une capitale d'empire et celles d'une capitale du peuple celtique, [...] est risquée, mais mérite d'être gardée en mémoire. » [35]. Passer alors de réunions restreintes ou de grands rassemblements pour y évoquer des sujets d'ordre collectifs ou communautaires est une chose, mais passer à des élections et substituer le rôle d'oppidum à celui de «monumental conciliabulum » sur la base de rouelles, de perles de verre (signes indubitables de jetons de vote selon l'auteur) et d'une analogie peu prudente avec les Saepta en est une autre. Ajoutons à cela que l'utilité des fossés eux-mêmes soit oubliée, laissant donc en suspens la proposition de « vide sanitaire » [36].

\section{LE VILLAGE AGRICOLE D'ACY-ROMANCE (ARDENNES)}

À Acy-Romance, les fouilles réalisées depuis les années 1990 au lieu-dit «La Warde » ont permis de caractériser la presque totalité d'un habitat groupé ouvert de 20 ha. Au cœur du village, sur le point culminant du plateau [37], se distingue une grande place de forme irrégulière en « $D$ » d'environ $4000 \mathrm{~m}^{2}$ (fig. 12). Cet espace central était délimité par une palissade continue constituée de poteaux jointifs et dont le prolongement interne dans l'angle sud-est a bien pu constituer l'unique accès [38]. Une série de bâtiments interprétés comme des temples borde la palissade occidentale de la grande place [39] et pourrait, comme à Corent, suggérer une éventuel lien fonctionnel Les seuls aménagements identifiés à l'intérieur de cet espace sont au nombre de six et prennent la forme de paires de poteaux parfaitement alignés et implantés à intervalles réguliers. Bien qu'aucun autre aménagement de la sorte n'ait encore été identifié dans le monde celtique, P. Méniel les interprète comme d'éventuelles potences utilisées dans le cadre de pratiques sacrificielles et bouchères [40]. Il signale également que le déroulement de telles pratiques, rassemblant à la fois des individus et des animaux « impose des contraintes matérielles, au premier rang desquelles celle de disposer d'un emplacement assez vaste » [41]. C'est à ce titre que la place d'Acy-Romance a pu accueillir ce type de concentrations humaines et animales.

\section{MODALITÉS D'IMPLANTATION ET D'ORGANISATION}

\section{DES ENSEMBLES CENTRAUX}

Les quatre exemples décrits précédemment mettent bien en évidence l'existence de grands espaces de superficies variables et généralement vides de construction.

[34] Ibid., p. 165.

[35] Dondin-PAYRE 2012, p. 62 ; I'excursus fait par I'auteur de cette citation mérite une grande attention et fait écho à un autre article, voir MARC 2011.

[36] Notons tout de même que les fouilles entreprises dès 2007 par $P$. Ruby pourraient apporter des précisions importantes quant aux propriétés et fonctions supposées des fossés. Pour l'heure, il semblerait que la piste explorée soit celle de «bassins-caissons », voir RUBY \& AUXIETTE 2011, p. 241.

[37] LAMBOt 2000, p. 22.

[38] Ibid., p. 27.

[39] LAMBOT 2006, p. 118.

[40] MÉNIEL 2001, p. 72.

[41] MÉNIEL 2008, p. 172. 
Quelle que soit la nature du site, la place semble systématiquement implantée de manière centrale, parfois sur un point culminant, de manière à jouer le rôle de point de convergence idéal de la communauté. Se développant face au sanctuaire monumental, I'esplanade de Corent se trouve au cœur du réseau urbain. Elle incarne le centre-ville autour duquel devaient s'organiser les îlots adjacents. Même observation pour le monde romain : le forum occupait généralement une situation privilégiée : «Si la ville est sur le bord de la mer, il faudra que I'endroit où I'on veut bâtir la place publique soit près du port ; tandis que, si la ville est éloignée de la mer, le forum devra se trouver au centre » (Vitruve, De Architectura, I, 7). Toutefois, il est important d'observer que la place subit les changements urbanistiques de la ville au même titre que les quartiers périphériques. La réorientation des espaces domestiques et artisanaux, ainsi que des grandes voies d'accès affectent les limites de la place, sa morphologie ainsi que sa surface au sol. Malgré cette mutation majeure, l'esplanade reste inoccupée et son emplacement inchangé tout au long de la période durant laquelle elle a été fréquentée. On constate donc qu'à Corent, la place possède un double statut, d'abord celui d'entité en parfaite adéquation avec le reste du tissu urbain, puis celui d'espace conservant une relative indépendance vis-à-vis des îlots qui la délimitent. À Acy-Romance et à Villeneuve-SaintGermain, la place occupant presque un demi-hectare de superficie et les $1250 \mathrm{~m}$ estimés de fossés sont tous deux situés au centre de l'habitat. Dans un cas la place est bordée par des quartiers spécialisés particulièrement bien structurés, son tracé, sa superficie ainsi que son organisation interne ne changent absolument pas, de son édification au démantèlement des palissades. Elle semble à la fois entretenir une relation étroite avec son environnement, tout en restant particulièrement «autonome » et « indépendante ». Dans un autre cas, les fossés font d'une part figures d'éléments de délimitation de I'habitat lui même, en le découpant en quatre quarts, et d'autre part de dispositif central donnant une cohérence unique à l'organisation de l'oppidum. Pour le Titelberg, le cas de figure est sensiblement différent. La dizaine d'hectares occupée par de rares aménagements est strictement isolée des quartiers qui se développent au-delà du fossé de clôture.

Une fois ce constat établi, il semble que deux grandes catégories de places se démarquent : une première parfaitement illustrée par l'exemple de Corent, où la place communique librement avec le reste du tissu urbain et une seconde, comme au Titelberg, où la place localisée en périphérie intra-muros se trouve isolée du reste de I'habitat.

\section{DES ENSEMBLES BIEN DÉLIMITÉS}

Ceci nous amène à évoquer les systèmes de clôture qui définissent les limites de ces espaces. Les dispositifs de délimitation sont variés et peuvent même être doublés afin d'accentuer la séparation. À ce jour, quartiers attenants, réseau viaire, fossés, palissades, levées de terre, murs en briques crues et même remparts, constituent les principales limites connues. Au Titelberg, l'espace est délimité par l'immense fossé qui interrompt le tracé de la voie principale et plus inédit encore, le rempart vient lui aussi faire office de clôture monumentale. À Acy-Romance, les limites sont clairement assurées par une palissade. Toutes ces limites témoignent d'une volonté d'asseoir l'existence physique de tels aménagements, voire d'une certaine recherche de mise en scène. À l'image de l'édification des remparts résultant d'une œuvre communautaire, il est tout à fait possible d'imaginer que ces systèmes de délimitation soient le fruit d'un véritable engagement communautaire. Pour Corent si les limites ne sont pas flagrantes, l'esplanade forme une enclave circonscrite à la fois par les rues et les quartiers environnants tout en se développant face au sanctuaire. Ici nous avons l'exemple parfait d'un aménagement communautaire inséré dans une trame urbaine impliquant des logiques d'implantation bien réglementées et faisant appel à une autorité publique. Même observation pour les grands fossés «en croix » de Villeneuve-Saint-Germain : ces derniers, pour reprendre les mots de J. Debord « ne sauraient résulter d'improvisation et témoignent de l'existence de spécialistes capables d'élaborer des projets architecturaux complexes qui répondent à la volonté de prestige d'un pouvoir politique suffisamment fort pour imposer la réalisation ».

\section{UNE VÉRITABLE VOLONTÉ DE « MISE EN SCÈNE 》}

Les opérations de terrassement peuvent aussi résulter d'un même désir de « scénographie » [42]. Par exemple à Corent, l'esplanade a fait l'objet d'une excavation jusqu'au substrat basaltique. Ce nivellement du terrain a eu comme principale conséquence d'entraîner la disparition des couches antérieures à l'époque laténienne. Le constat est le même pour le complexe $C$. Son édification a elle aussi été précédée d'une mise à nu du substrat. Tout cela confère au sol un aspect plus soigné, moins rudimentaire ainsi qu'un usage plus adapté à la circulation.

Le choix de l'emplacement est lui aussi un gage de mise en valeur et de monumentalisation. Dans la plupart des cas, tous ces espaces sont implantés de manière à occuper une place centrale sur le site [43].

[42] Poux 2007, p. 113.

[43] FICHTL 2012, p. 50. 
Cela est particulièrement frappant à Acy-Romance où la place a été implantée sur le point culminant du plateau, tout en adoptant la topographie du paysage. La portion curviligne du fossé suit ainsi précisément l'ultime courbe de niveau. Au Titelberg, le constat est le même. La grande halle à trois nefs est édifiée de manière à dominer l'ensemble de la ville. Elle est à la fois construite sur le point le plus élevé du plateau, mais aussi parfaitement inscrite dans l'axe des deux portes d'accès de l'agglomération trévire. Une fois celles-ci franchies, la halle bénéficiait d'une visibilité sans pareil pour toute personne entrant dans I'oppidum [44]. La dimension scénographique est tout aussi bien marquée à Corent par la situation centrale qu'occupe l'esplanade. Vide de structures, elle se situe d'une part dans le prolongement exact du sanctuaire et offre, d'autre part, un point d'observation indéniable en direction de sa tour-porche [45].

La surface occupée par ces espaces, couplée à l'absence de vestiges de constructions, représente en soi un argument de choix pour avancer I'hypothèse d'une véritable volonté de mise en scène. Ces deux caractéristiques renforcent ainsi l'identité de ces espaces se définissant « en négatif », par opposition aux quartiers périphériques plus densément aménagés.

\section{UNE FONCTION SOCIALE?}

$\mathrm{Au}$ regard de tous ces éléments, il semble que ces espaces devaient « mélanger » une multitude d'activités et de pratiques. Hormis des usages artisanaux, économiques, politiques ou encore religieux, leur dimension sociale ne doit pas être sous-estimée.

Pour cette période, l'archéologie peut difficilement apporter des preuves tangibles validant ou non cette hypothèse. Plusieurs travaux peuvent néanmoins servir de point de départ à cette réflexion : d'une part, les études anthropologiques de P. Bohannan et de G. Dalton abordant les « aspects non économiques des places de marchés » [46] et d'autre part, les multiples travaux sur les agorai grecques et les fora romains. Ces lieux de vie collective incarnent ce que I'on pourrait appeler des espaces de «socialisation ». Ils pouvaient en effet aisément permettre aux gens d'échanger et d'entretenir des relations avec des étrangers, des parents ou des amis. Dès lors, il est possible d'imaginer à quel point ce véritable nœud de communication permettait tout aussi bien de s'informer que d'informer, de faire circuler certaines nouvelles ou d'en prendre connaissance.

[44] MetZler et al. 2006, p. 204.

[45] Poux 2012, p. 31

[46] BohanNan \& DaLTON 1962, p. 15-19.
Pour reprendre une citation de J. Le Gall à propos du forum romain : « [...] le forum était l'endroit le plus fréquenté de la ville; on y venait même quand on n'avait pas de raison particulière de $s^{\prime} y$ rendre, simplement pour y rencontrer d'autres gens, apprendre les nouvelles, faire le badaud, passer le temps au besoin avec des petits jeux dont on dessinait le schéma sur les dalles, pour voir et $s^{\prime} y$ faire voir : on « s'habillait » pour y aller » [47].

\section{CONCLUSION}

À l'issue de cette étude, l'espace de vente gaulois nous apparaît bien plus complexe qu'il n'y paraît. Exception faite de l'ensemble sur cour de Corent, les lieux spécialisés dans les échanges marchands devaient être très minoritaires, voire presque inexistants. Aucun autre exemple n'a en effet démontré l'existence d'aménagements dont la nature et la fonction soient aussi significatives.

Dès lors, se limiter à une réflexion uniquement portée sur les lieux de commerce paraît compliqué, même impossible. Au regard des récentes données, il semble envisageable d'identifier de grands espaces plurifonctionnels dont les activités commerciales et de production ne devaient être qu'un aspect parmi d'autres. Si dans l'imaginaire collectif, agorai et fora incarnent l'excellence de l'espace public, pouvons-nous invoquer à l'appui des recherches récentes l'existence d'ouvrages d'usage collectif et communautaire pour le monde gaulois ? C'est en tout cas ce que les découvertes archéologiques tendent à prouver dans certains habitats groupés de La Tène moyenne et finale. Cela apporterait une possible explication à I'un des passages du Bellum Gallicum, où César trouva utile de mentionner lors du siège de BourgesAvaricum que les insurgés Bituriges se rassemblèrent «sur le forum et dans les lieux les plus ouverts » [48].

En guise d'ouverture ne manquons pas de mentionner I'esplanade de Batilly-en-Gâtinais (Loiret) qui occupe plus d'un tiers du site et se développe face à une tour-porche. Cette installation vient mettre en perspective notre réflexion sur les espaces publics en nous interpellant sur la propriété et les usages d'un tel aménagement au sein d'un habitat à caractère privé. Pour I'heure, S. Fichtl suppose qu'en l'absence d'oppida ou d'agglomérations importantes dans la région, cette esplanade a pu jouer le rôle de grand lieu de commerce pour la redistribution de marchandises et de denrées agricoles [49]. N'oublions pas, tout de même, qu'en raison de la nature même du

[47] Le Gall 1978, p. 25.

[48] CÉSAR, La guerre des Gaules, VII, 28.

[49] FICHTL 2009, p. 47. 
site l'un de ses usages devait être agropastoral comme I'attestent les outils, les meules, les structures de stockage et autres annexes retrouvés contre la bordure extérieure des palissades.

Peut-être que I'historien qu'était C. Jullian n'avait pas tout à fait tort en proposant l'existence de vastes « lieux de sociabilité »[50] : «Semblable en cela à la France du Moyen Âge, la Gaule fut peut-être le pays de I'Antiquité qui a le plus créé de ces rendez-vous de foires : elle aimait les larges aires découvertes, propres aux entassements familiers de tentes, de bestiaux, de denrées, d'êtres humains, ces solennelles assises sociales, bruyantes et gaies, où se mêlent les complots, les disputes, les chants, les prières et les nouvelles, et où du contact de tant d'hommes naît l'agitation de toutes les facultés, et cette ivresse de la vie que recherchait la race gauloise. Car il faut se représenter ces champs de marchés comme ceux de l'ancienne France ou les lieux des pardons de la Bretagne actuelle : d'un côté, les endroits sacrés, autel ou temple, source ou fontaine, arbres ou bosquet; de I'autre, l'esplanade où l'on se rassemble ; plus loin, celle où I'on campe; les réunions d'affaires se tenant à la veille ou au lendemain des jours de fête ; les prêtres coudoyant les marchands, la foire alternant avec le pèlerinage, les dieux se taillant leurs parts dans les profits des hommes »[51].

[50] Audouze \& BuchSEnschutz 1989, p. 188.

[51] JULLIAN 1964, p. 239.

Allen, Tim, Biddulph, Edward, Dodd, Mark, Donnelly, Mike, Gourlin, Bertrand, \& Poole, Cynthia, 2012, Rocade d'agglomération briochine (Trégueux - Côtes-d'Armor - Bretagne). Enclos défensif, bâtiment public et habitat nucléé de La Tène 2, et leurs développements à la période gallo-romaine et à l'époque médiévale, Rapport de fouilles, SRA Rennes.

Audouze, Françoise \& Buchsenschutz, Olivier, 1989, Villes, villages et campagnes de l'Europe celtique : du début du II millénaire à la fin du $I^{\mathrm{er}}$ siècle avant J.-C., Paris.

Blein, Christine, 2012, «L'implantation des lieux de vente dans les centres des villes de Grèce du Nord à la fin de l'époque hellénistique et à l'époque impériale », dans V. Chankowski \& P. Karvonis (dir.), Tout vendre, tout acheter. Structures et équipements des marchés antiques, Actes du colloque international 2-4 novembre 2009, Bordeaux, p. 13-18.

Bohannan, Paul \& Dalton, George (dir.), 1962, Markets in Africa, Northwestern University Press.

Buchsenschutz, Olivier, 2008, Les Celtes, Paris.

Chankowski, Véronique \& Karvonis, Pavlos, 2012, « Introduction », dans V. Chankowski \& P. Karvonis (dir.), Tout vendre, tout acheter. Structures et équipements des marchés antiques, Actes du colloque international 2-4 novembre 2009, Bordeaux, p. 13-18.

Debord, Jean, 1990, «Les fouilles du site gaulois tardif de Villeneuve-Saint-Germain (Aisne) », Mémoires de la fédération des sociétés d'histoire et d'archéologie de l'Aisne 35, p. 137-170.

Debord, Jean, Lambot, Bernard \& Buchsenschutz, Olivier, 1988, « Les fossés couverts du site gaulois tardif de VilleneuveSaint-Germain (Aisne) », Archéologie aujourd'hui. Dossiers de Protohistoire 2, p. 121-135.

Demierre, Matthieu \& Poux, Matthieu, 2012, «Du cultuel au profane : essai d'analyse taphonomique et spatiale des petits mobiliers du sanctuaire de Corent et de ses abords », dans O. De Cazanove \& P. Méniel (dir.), Étudier les lieux de culte de Gaule romaine, Actes de la table-ronde de Dijon 18-19 septembre 2009, Montagnac, p. 209-227. 
Descat, Raymond, 2012, « Avant-propos », dans V. Chankowski \& P. Karvonis (dir.), Tout vendre, tout acheter. Structures et équipements des marchés antiques, Actes du colloque international 2-4 novembre 2009, Bordeaux, p. 11-12.

Dondin-Payre, Monique, 2012, « Forum et structures civiques dans les Gaules : les témoignages écrits », dans A. Bouet (dir.), Le forum en Gaule et dans les régions voisines, Bordeaux, p. 55-63.

DuRkheim, Émile, (1960) [1912], Les formes élémentaires de la vie religieuse : le système totémique en Australie, Paris.

FichtL, Stephan, 2005, La ville celtique : Les oppida de 150 avant J-C à 15 après J.-C., Paris.

FichtL, Stephan, 2009, « Une proto-villa de la fin de l'époque gauloise », L'archéologue 95, p. 42-47.

FichtL, Stephan, 2012, «Places publiques et lieux de rassemblement à la fin de l'âge du Fer dans le monde celtique », dans A. Bouet (dir.), Le forum en Gaule et dans les régions voisines, Bordeaux, p. 41-53.

FichtL, Stephan, 2013, «Les agglomérations gauloises à la fin de l'âge du Fer en Europe celtique (III ${ }^{\mathrm{e}} \mathrm{I}^{\mathrm{er}}$ siècle av. J.-C.) 》, dans D. Garcia (dir.), L'habitat en Europe celtique et en méditerranée préclassique - Domaines urbains, Paris.

Fichtl, Stephan, Cony, Alexandra, Wassong, Rémy, Hutin, Thomas \& Pradier, Hubert, 2014, Batilly-en-Gâtinais/Boynes (Loiret) Résidence aristocratique gauloise $I I^{e}-I^{e r}$ s. av. J.-C., Rapport de fouilles, SRA Orléans.

Hutin, Thomas, 2014, Le problème de la caractérisation archéologique des lieux de l'échange dans la Protohistoire d'Europe occidentale $\left(I I I^{e}-I^{e r}\right.$ s. av. J.-C.), Mémoire de Master, Université Bordeaux Montaigne, Bordeaux.

Ginouvès, René, 1993, La Macédoine. De Philippe II à la conquête romaine, Paris.

Guichon, Romain, 2006, «Les rondelles en céramique du sanctuaire de Corent (Puy-de-Dôme) », SFECAG, Actes du Congrès de Pézenas, p. 517-524.

Jullian, Camille, 1964 [1907], Histoire de la Gaule. La Gaule indépendante, 2, Bruxelles.

LAмвот, Bernard, 2000, « Le village d'Acy-Romance dans son contexte régional », dans S. Verger (dir.), Rites et espaces en pays celte et méditerranéen. Étude comparée à partir du sanctuaire d'Acy-Romance, Rome, p.7-139.

LAмвот, Bernard, 2006, «Religion et habitat. Les fouilles d'Acy-Romance », dans Chr. Goudineau (dir.), Religion et société en Gaule, Paris, p. 177-190.

Le GaLl, Joël, 1978, «Que faisait-on sur le forum ? », dans M. Roland, J. Le Gall \& R. Chevalier (dir.), Forum et Plaza Mayor dans le Monde Hispanique, Colloque interdisciplinaire -Casa de Velázque z-Madrid 28 octobre 1976, Paris, p. 7-21.

Maguer, Patrick, 2007, «Le site aristocratique des Natteries, Le Puy-Saint-Bonnet (Maine et Loire) », dans I. Bertrand \& P. Maguer (dir.), De pierre et de terre. Les Gaulois entre Loire et Dordogne, catalogue de l'exposition présentée par les musées de la ville de Chauvigny (Vienne) du 15 mai au 14 octobre 2007, Donjon de Gouzon, Chauvigny, p. 78-90.

MARC, Jean-Yves, 2011, «Un excès de la romanisation ? L'identification dans les villes gauloises de monuments civiques romains », dans M. Reddé, P. Barral, F. Favory, J.-P. Guillaumet, M. Joly, J.-Y. Marc, P. Nouvel, L. Nuninger \& Chr. Petit (dir.), Aspect de la Romanisation dans l'Est de la Gaule, Glux-en-Glenne, p. 309-317.

MARTIN, Roland, 1978, « Agora et forum : ancêtres de la Plaza Mayor ? », dans R. Martin, J. Le Gall \& R. Chevalier (dir.), Forum et Plaza Mayor dans le Monde Hispanique, Colloque interdisciplinaire - Casa de Velázquez Madrid 28 octobre 1976, Paris, p. 7-21.

Menez, Yves, 2009, Le Camp de Saint Symphorien à Paule (Côtes d'Armor) et les résidences de l'aristocratie du Second âge du Fer en France septentrionale, Thèse de doctorat, Université de Paris 1, Panthéon-Sorbonne.

MénIel, Patrice, 2001, Les gaulois et les animaux. Elevage, repas et sacrifice, Paris.

MénIeL, Patrice, 2008, «Les restes d'animaux de l'espace public de I'oppidum du Titelberg », dans D. Castella \& M.-F. Krause (dir.), Topographie sacrée et rituels. Le cas d'Aventicum, capitale des Helvètes, Actes du colloque international 2-4 novembre 2006, Basel, p. 167-173.

Merlin, Françoise \& ChoAy, Pierre, 2005, Dictionnaire de l'urbanisme et de l'aménagement, Paris.

MetzleR, Jeannot, 1991, «Les sanctuaires gaulois en territoire trévire», dans J.-L. Brunaux (dir.), Les sanctuaires celtiques et leurs rapports avec le monde méditerranéen, Actes du colloque de St-Riquier, 8-11 novembre 1990, Paris, p. 28-41.

MetzleR, Jeannot, 2008, «Du Titelberg à Trèves. De I'oppidum gaulois à la ville romaine », dans D. Castella \& M.-F. Krause (dir.), Topographie sacrée et rituels. Le cas d'Aventicum, capitale des Helvètes, Actes du colloque international 2-4 novembre 2006, Basel, p. 155-165.

Metzler, Jeannot, Gaeng, Catherine \& Méniel, Patrice, 2006, «Religion et politique. L'oppidum trévire du Titelberg», dans Chr. Goudineau (dir.), Religion et société en Gaule, Paris, p. 191-202.

Metzler, Jeannot, Méniel, Patrice \& Gaeng, Catherine, 2006, «Oppida et espaces publics 》, dans C. Haselgrove (dir.), Les mutations de la fin de l'âge du Fer, Actes de la table ronde de Cambridge 7-8 juillet 2005, Glux-en-Glenne, p. $201-224$.

Nouvel, Pierre \& Barral, Philippe, 2014, « La partie occidentale des terrasses PC 14 et PC 15 intervention au pied oriental du Theurot de la Roche », Bibracte. Programme quadriennal de recherche 2013-2016 sur le Mont-Beuvray, Rapport de fouilles, Glux-en-Glenne, p. 93-145.

Peyre, Christian, 2000, «Documents sur l'organisation publique de l'espace dans la cité gauloise. Le site de Villeneuve-SaintGermain et la bilingue de Verceil », dans S. Verger (dir.), Rites et espaces en pays celte et méditerranéen, Rome, p. 155-206.

Poux, Matthieu, 2006, Corent. Oppidum et sanctuaire (II), Rapport de la campagne 2006, Rapport de fouille, SRA Clermont-Ferrand.

Poux, Matthieu, 2007, Corent. Oppidum et sanctuaire (III), Campagne 2007 - Rapport final d'opération, Rapport de fouille, SRA Clermont-Ferrand.

Poux, Matthieu, 2008, Corent. Rapport de fouilles 2008, Rapport de fouille, SRA Clermont-Ferrand.

Poux, Matthieu, 2012, Corent : voyage au cœur d'une ville gauloise, Paris.

Ruby, Pascal \& Auxiette, Ginette, 2011, Oppidum du deuxième âge du Fer de Villeneuve-Saint-Germain (Aisne). Projet de fouilles pluriannuelles programmées 2007-2010. Rapport final programme triennal, Rapport de fouilles, Soissons.

Ruby, Pascal \& Auxietre, Ginette, 2013, Fouilles sur l'oppidum du second âge du Fer de Villeneuve-Saint-Germain. Rapport de la première année complémentaire du programme triennal 2007-2010, Rapport de fouilles, Soissons. 